\title{
BMJ Open British Thoracic Society survey of rehabilitation to support recovery of the post-COVID-19 population
}

\author{
Sally J Singh, ${ }^{1,2}$ Amy C Barradell (D , , ${ }^{1,2}$ Neil J Greening, ${ }^{1,2}$ Charlotte Bolton, ${ }^{3}$ \\ Gisli Jenkins, ${ }^{3}$ Louise Preston, ${ }^{4}$ John R Hurst (D) ${ }^{5}$
}

To cite: Singh SJ, Barradell AC, Greening NJ, et al. British Thoracic Society survey of rehabilitation to support recovery of the post-COVID-19 population. BMJ Open 2020;10:e040213. doi:10.1136/ bmjopen-2020-040213

- Prepublication history and additional material for this paper is available online. To view these files, please visit the journal online (http://dx.doi.org/10. 1136/bmjopen-2020-040213).

Received 07 May 2020 Revised 21 October 2020 Accepted 31 October 2020

A) Check for updates

(c) Author(s) (or their employer(s)) 2020. Re-use permitted under CC BY-NC. No commercial re-use. See rights and permissions. Published by BMJ.

${ }^{1}$ Centre for Exercise and Rehabilitation Science, NIHR Leicester Biomedical Research Centre-Respiratory, University Hospitals of Leicester NHS Trust Leicester, UK

${ }^{2}$ Department of Respiratory

Sciences, University of Leicester, Leicester, UK

${ }^{3} \mathrm{NIHR}$ Nottingham Biomedical Research Centre Respiratory Theme, School of Medicine, University of Nottingham, Nottingham, UK

${ }^{4}$ Communications, Education and Quality Improvement, British Thoracic Society, London, UK ${ }^{5}$ UCL Respiratory, University College London, London, UK

Correspondence to

Dr Sally J Singh;

sally.singh@uhl-tr.nhs.uk

\section{ABSTRACT}

Objective A proportion of those recovering from COVID-19 are likely to have significant and ongoing symptoms, functional impairment and psychological disturbances. There is an immediate need to develop a safe and efficient discharge process and recovery programme. Established rehabilitation programmes are well placed to deliver a programme for this group but will most likely need to be adapted for the post-COVID-19 population. The purpose of this survey was to rapidly identify the components of a post-COVID-19 rehabilitation assessment and elements of a successful rehabilitation programme that would be required to deliver a comprehensive service for those postCOVID-19 to inform service delivery.

Design A survey comprising a series of closed questions and a free-text comment box allowing for a qualitative analysis.

Setting Online survey.

Participants Multiprofessional clinicians across

specialties were invited to take part.

Results 1031 participants responded from a broad range of specialties. There was overwhelming support for an early posthospital discharge recovery programme to advise patients about the management of fatigue (95\% agreed/strongly agreed), breathlessness (94\%) and mood disturbances (including symptoms of anxiety and depression, 92\%). At the time point of 6-8 weeks, an assessment was considered important, focusing on a broad range of possible symptoms and supporting a return to work. Recommendations for the intervention described a holistic programme focusing on symptom management, return of function and return to employment. The free-text comments added depth to the survey and the need 'not to reinvent the wheel' but rather adapt well-established rehabilitation services to individually tailor needs-based care with continued learning for service development. Conclusion The responses indicate a huge interest and the urgent need to establish a programme to support and mitigate the long-term impact of COVID-19 by optimising and individualising existing rehabilitation programmes.

\section{BACKGROUND}

Since December 2019, the global COVID-19 pandemic has already resulted in tens of thousands of people being admitted to the hospital for acute medical management, a proportion of whom will have had a prolonged stay in

\section{Strengths and limitations of this study}

- Large and comprehensive survey conducted to guide the provision of post-COVID-19 assessment and rehabilitation.

- The survey provides clear recommendations for the provision of advice and support immediately on discharge.

- The survey provides recommendations for a programme of holistic rehabilitation 6-8 weeks postdischarge based on the existing rehabilitation models.

- Seventy-one per cent of respondents were physiotherapists and $84 \%$ were female, limiting the generalisability of results to all relevant specialties.

- Twenty-five per cent of respondents had no experience in managing patients with COVID-19, and $31 \%$ had no experience in rehabilitation.

intensive care units (ICUs). Those discharged from ICUs are likely to exhibit significant ongoing symptoms, notably, dyspnoea, fatigue and cough, functional impairment and psychological disturbances. ${ }^{1-3}$ The larger cohort of people discharged after ward-based care or managed in the community are also likely to experience similar problems.

Although we have limited COVID-19 data so far, literature from the SARS outbreak would suggest that there is a considerable impact on the individual with reduced functional performance and health status even at 6 months postdischarge compared with normal values. ${ }^{45}$

There is a pressing need to develop a safe and efficient discharge process to support patients in the early phase of recovery and to set up a mechanism to review these individuals early in the postdischarge phase to facilitate care planning, onward referral, restoration of premorbid function, holistic well-being, and participation in family, community and work life. Furthermore, any pathway should be accessible to those who remained in the 
community to manage their COVID-19 infection but have had a slow and incomplete recovery.

There are existing rehabilitation pathways that assess and manage the rehabilitation needs of patients with long-term conditions, notably, cardiac and pulmonary rehabilitation pathways. These services accommodate patients with multiple comorbidities, including chronic respiratory disease, and cardiovascular, mental health and metabolic diseases. ${ }^{67}$ There is a strong evidence base demonstrating that a centre-based, supervised outpatient programme of education and physical activity impacts on symptom burden, for example, breathlessness, anxiety, depression, health status and exercise capacity. As an example, pulmonary rehabilitation is an interdisciplinary intervention that integrates a broad group of healthcare professionals including, but not limited to, physiotherapists, nurses, dietitians, pharmacists, psychologists, physicians, occupational therapists, exercise physiologists and graduates of the programme. The provision of pulmonary rehabilitation is demonstrably successful in clinical practice outside the context of research studies. UK data from over 7000 cases have been collected and reported as part of the National Asthma and COPD Audit Programme (Pulmonary Rehabilitation). ${ }^{8}$ Furthermore, patients frequently have multiple long-term conditions that do not compromise the outcome, the common comorbidities recorded in the chronic respiratory population mirror, those that have been reported in the COVID-19 population of hypertension, diabetes and cardiovascular disease. ${ }^{9}{ }^{10}$ Additionally, a recent review proposed that a referral to a pulmonary rehabilitation programme for the individual post-COVID-19 who remains symptomatic is appropriate. ${ }^{11}$

However, the rehabilitation needs of the postCOVID-19 population are likely more diverse than those commonly observed in pulmonary and cardiac rehabilitation programmes. Early data from Wuhan in China indicate that the mean age of people hospitalised with COVID-19 was 52 (45-58) years ${ }^{9}$ compared with 69 (60-78) years reported for a conventional pulmonary rehabilitation programme. ${ }^{12}$ However, data from the UK International Severe Acute Respiratory and Emerging Infection Consortium registry of 16749 COVID-19 admissions indicate that the median age is $72(57-82)$ years, ${ }^{13}$ more typical of the pulmonary rehabilitation population. However, given the widespread nature of the pandemic, there will be a substantial number of younger patients, including those admitted to ICU, and in some of the patients post-COVID-19, their premorbid state is likely to be quite different. Many may not have pre-existing lung disease, and likely different levels of employment, usual levels of activity and exercise behaviours. Furthermore, there is an indication the post-COVID-19 population is likely to have significant psychological and cognitive impairments, particularly if management involved a stay in ICU. ${ }^{14}$ There is some evidence indicating that pulmonary rehabilitation interventions in the SARS population and acute respiratory distress syndrome are effective. ${ }^{15}$
We postulated that while the core of pulmonary rehabilitation would in part meet the needs of the patient post-COVID-19, the programme would likely need to be adapted. The modifications would primarily be at the point of assessment to broaden the scope to holistically assess the impact of COVID-19 and, second, to address the components of a comprehensive programme which considers the psychological and mental health needs of patients in recovery.

Therefore, the purpose of this survey was to rapidly identify the additional components of a post-COVID-19 rehabilitation assessment and elements of a successful rehabilitation programme that would be required to deliver a comprehensive service for those either discharged from the hospital post-COVID-19 or for those managed in the community with marked ongoing symptoms that prevent a full recovery.

\section{METHODS}

We conducted a survey of multiprofessional clinicians. The survey was designed in collaboration with the British Thoracic Society (BTS) and a team with expertise in pulmonary and cardiac rehabilitation and the wider management of respiratory disease. The survey was predominately composed of closed questions, with a freetext box at the end of the survey for additional comments. The survey was built by the team at the BTS using ClassApps software. The survey was tested by local teams experienced in rehabilitation prior to the wider launch.

The initial stages of the survey asked for basic demographic information from the participants to include age, gender, ethnicity, professional background, location of work and exposure to patients with or recovering from COVID-19 (the full survey is available in online supplemental file 1).

The purpose was to gain wide clinical consensus as to what an effective, holistic rehabilitation intervention might comprise for patients recovering from COVID-19. The survey aimed to secure guidance for rehabilitation support provided in two phases:

- The initial discharge period (which may be to home, a step-down unit or a rehabilitation facility).

- A formal rehabilitation programme that would be offered 6-8 weeks postrehabilitation. This time period is based on evidence accumulated by an ad hoc task force formed by the American Thoracic Society/ European Respiratory Society with a supporting document. ${ }^{16}$

For these sections of the survey, there was a statement and participants were invited to respond with the following five categories: 'strongly disagree', 'disagree', 'neutral', 'agree' or 'strongly agree'. There was also the option to respond with 'unable to comment'.

On completion of the survey, there was an additional free-text box for further comments. No questions were mandatory. 


\section{Patient and public involvement}

As the survey was directed towards healthcare professionals, there was no patient or public involvement.

\section{Survey distribution}

The survey was available to participants from 9 April 2020 to 15 April 2020. Seven days' access covered a bank holiday, scheduled workdays and a weekend which maximised opportunities to complete the survey. It was distributed to members of the BTS via the societies' e-newsletter and healthcare professionals via the BTS Twitter account. A reminder email was sent to BTS members 6 days later; a reminder to participate was retweeted by society members and the BTS encouraging both BTS members and healthcare professionals to participate and to share the survey with colleagues. The survey was not restricted to UK-based healthcare professionals, although the country of practice was noted on the survey.

Participation in the survey was voluntary and anonymous. Participants confirmed their willingness to engage in this research by accessing and completing the online survey.

\section{Data analysis}

Quantitative data were reported as counts and percentages for each category of the demographic and survey responses. At least $70 \%$ agreement on directionality (combining strongly agree and agree) was defined as the threshold for consensus.

Qualitative data were analysed using thematic analysis. ${ }^{17}$ The text data were uploaded to NVivo V.12 Pro and then coded and grouped into themes to portray patterns within the data. The established themes were reviewed by the first author and the finalised themes were defined.

Completion of the survey was an indication of willingness to participate and implied consent. We set no threshold for response over such a short period of time but were anticipating around 300 responses across a range of healthcare professionals to allow the questionnaire to be considered robust and representative of those in the field.

\section{RESULTS}

This report is based on data from 1031 respondents. A further 750 respondents only provided answers to the demographic questions on page 1 and therefore do not form part of this report; however, their demographics are consistent with the results presented as follows. The majority of respondents were female $(84 \%)$; the largest age group was $35-44$ years $(34 \%)$, followed by $45-54$ years (27\%) and 25-34 years (22\%). A significant majority identified as white British (75\%), with any other white background, white Irish and Indian representing 7\%, 6\% and $5 \%$ of the participants, respectively. The largest group of respondents were from England $(80 \%)$, with Scotland representing $6 \%$ of respondents, Wales $4 \%$, Northern Ireland $3 \%$ and the remainder from the rest of Europe,
Australasia and South America. Respiratory (32\%) represented the largest known group by specialism, followed by healthcare of the elderly (12\%), primary care $(7 \%)$, acute medicine $(6 \%)$, and sport and exercise $(5 \%)$, with smaller numbers from cardiology, general medicine, anaesthetics, psychiatry and psychology backgrounds. However, 34\% recorded 'other' backgrounds, which included neurology $(n=44)$, critical care $(n=26)$ and musculoskeletal $(n=56)$. Physiotherapists represented the largest group $(71 \%)$; dietitians, nurses and consultant physicians represented $7 \%, 6 \%$ and $6 \%$, respectively; smaller numbers of occupational therapists, speech and language therapists, trainee physicians and healthcare assistants participated. The majority worked in either secondary care hospital (45\%) or community hospitals/ services (28\%), with fewer responses from either primary care or private hospitals; $17 \%$ of 'others' were represented by the private (industry) sector, universities, the community (excluding hospitals) and hospices. The final two profiling questions allowed for more than one response; in total, there were 1420 responses as to whether or not they had experience in managing patients with COVID19. A total of 361 (25\%) respondents had no experience; the remainder had experience on acute wards $(n=332$, $23 \%)$, ICUs $(n=257,18 \%)$, community $(n=209,15 \%)$ and step-down units $(\mathrm{n}=154,11 \%)$. With respect to rehabilitation, $442(31 \%)$ respondents had no experience; 216 $(15 \%)$ had experience in pulmonary rehabilitation; 208 $(15 \%)$ had experience in healthcare of the elderly; 52 (4\%) had experience in cardiac rehabilitation; and 202 $(14 \%)$ had experience in other forms of rehabilitation. Of the 1030 respondents, 167 (16\%) had no experience in managing patients with COVID-19 or rehabilitation.

\section{Recommendations for the early phase of COVID-19 recovery}

The first section of the survey addressed the immediate postdischarge phase, that is, care or advice delivered in the home, in a step-down unit or in a rehabilitation hospital/ ward. Items that reached the threshold for recommendation for the early-phase recovery programme are displayed in figure 1. All but one proposed survey items (online/digital delivery) were recommended for the early phase of COVID-19 recovery. There was overwhelming support for early postdischarge from the hospital phase of the recovery programme to advise patients about the management of fatigue (95\% agreed or strongly agreed), breathlessness $(94 \%)$ and mood disturbances (including symptoms of anxiety and depression, 92\%). In recognition of the current UK community 'lockdown', there was clear agreement to provide support for coping with social isolation (91\%). At this early stage in the recovery process, there were less strong recommendations about cough management, delivery of an exercise programme or support for post-traumatic stress disorder (PTSD), but these comfortably exceeded the $70 \%$ threshold, at $81 \%$, $80 \%$ and $78 \%$, respectively. Advice provided on a digital platform failed to reach the $70 \%$ threshold (59\%), with 


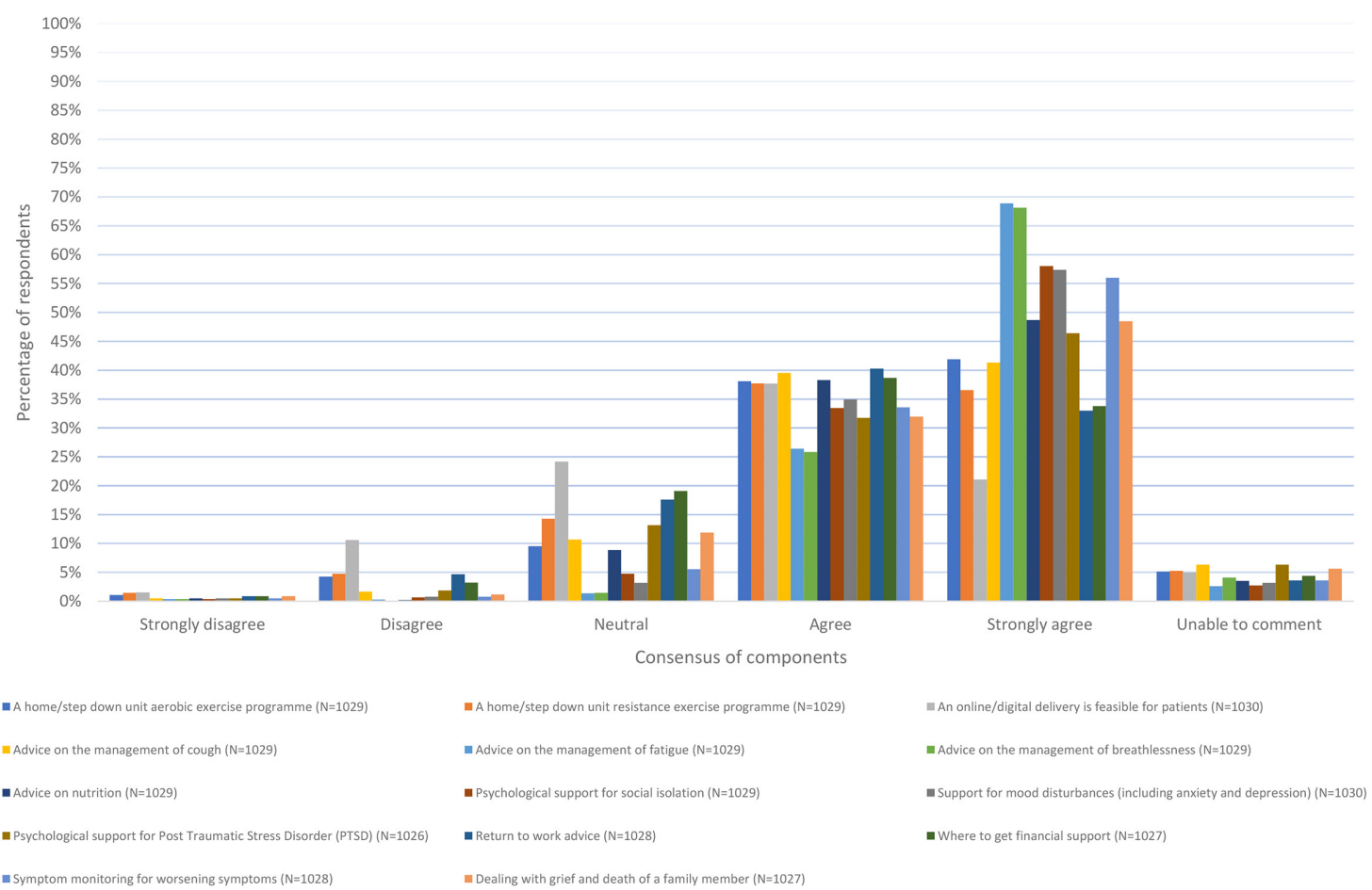

Figure 1 Essential components of an early-phase recovery programme (first few weeks after discharge/episode).

$24.2 \%$ being neutral. This question provided the largest 'neutral response'.

\section{Recommendations for assessment at 6-8 weeks posthospital discharge}

The essential components that reached consensus of an assessment at 6-8 weeks postepisode/hospital (or stepdown unit) discharge are displayed in figure 2 . There was strong support for the assessment of mood (93\% strongly agreed or agreed), quality of life (92\%) and fatigue (92\%). The assessment of cough just reached the $70 \%$ threshold with $71 \%$ recommending assessment. Advice with respect to returning to work (73\%) and financial support (72\%) were not rated as highly but also exceeded the $70 \%$ threshold. The items which did not reach consensus were the need for a face-to-face assessment, assessment of exercise capacity/muscle strength and the need for a measure

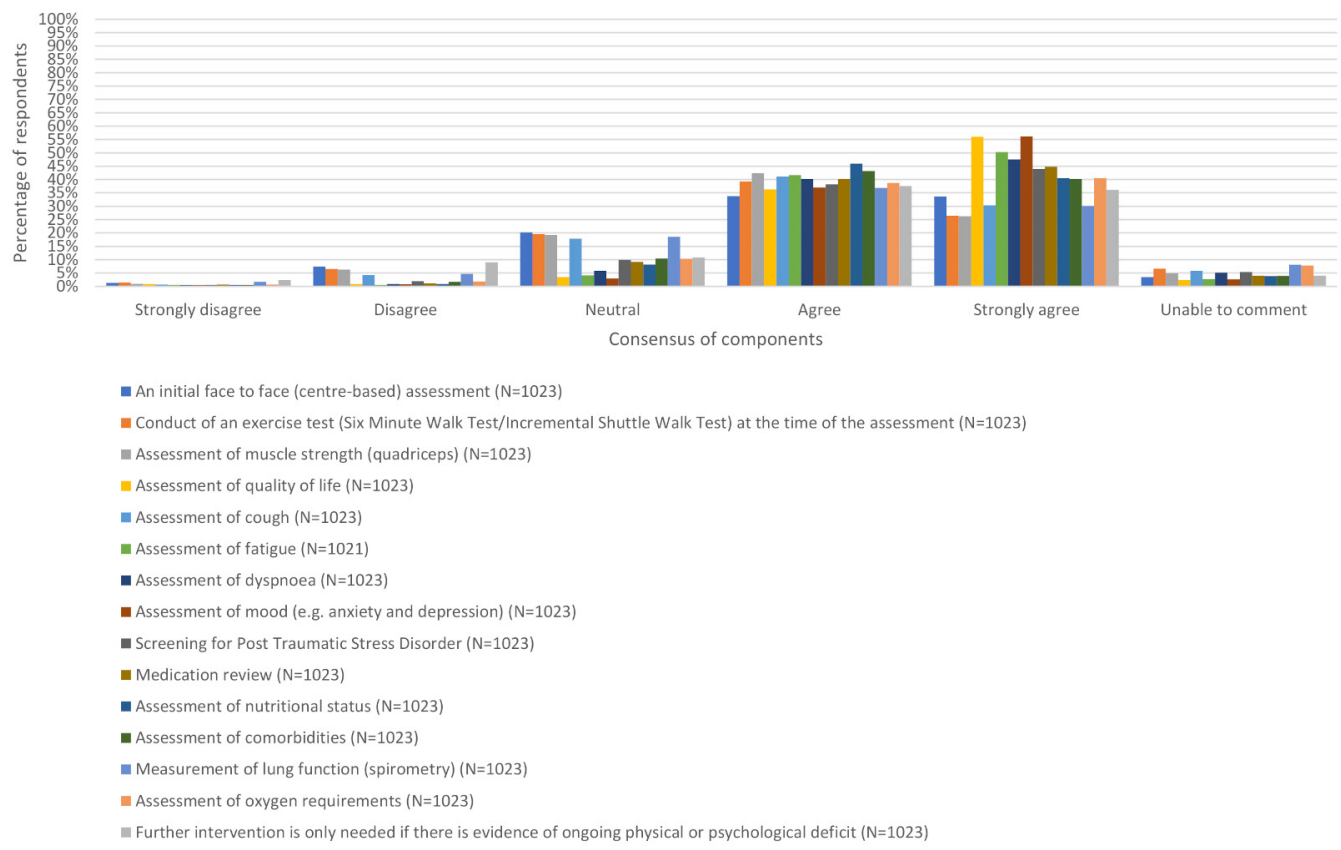

Figure 2 Essential components of an assessment at 6-8 weeks of posthospital (step-down unit) discharge. 

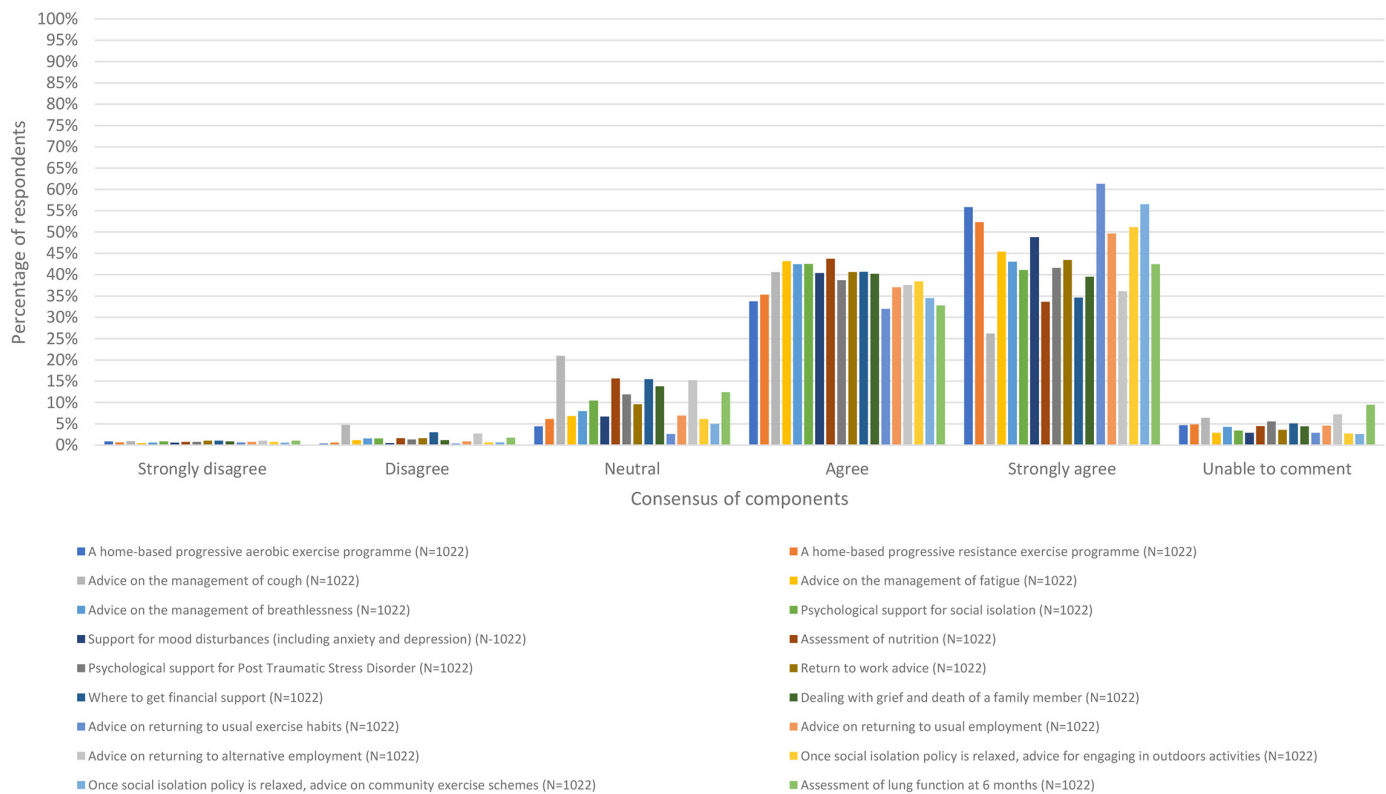

Figure 3 Essential components of a continued recovery programme beyond 6 weeks of posthospital (step-down unit) discharge.

of lung function with $68 \%, 66 \%$ and $69 \%$ of the survey participants, respectively, recommending these factors as an important part of the assessment.

\section{Recommendations for the components of a rehabilitation recovery programme for COVID-19}

The essential components which reached consensus for the later phase of recovery (6-8 weeks postdischarge/ episode and following the assessment outline earlier) are displayed in figure 3 . The most frequently recommended items included advice on returning to usual exercise habits (93\% either strongly agreeing or agreeing), $91 \%$ recommending advice on community exercise schemes, and, given the lockdown at the time of writing, advice on community exercise schemes (once social isolation policy is relaxed) and advice for engaging in outdoors activities (once social isolation policy is relaxed) were highly rated (91\% and $93 \%$ respectively) by respondents. Similarly, exercise advice for home-based aerobic and resistance programmes was highly rated $(90 \%$ and $88 \%$, respectively). Symptom management was rated, with advice on the management of fatigue and support for mood disturbances (including anxiety and depression) being equally strongly recommended at $89 \%$. Advice on the management of breathlessness was marginally less at $86 \%$. Advice on the management of cough did not reach the $70 \%$ threshold at $6-8$ weeks postdischarge, with the largest number of respondents reporting to be neutral for this question compared with any other question in this particular section $(21 \%)$. The impact on employment was also rated highly, including advice on returning to usual employment (87\%), where to get financial support advice $(75 \%)$ and advice on returning to alternative employment (74\%). Support for some unique aspects of COVID-19 and the current lockdown were also rated highly, including psychological support for social isolation $(84 \%)$, dealing with grief and death of a family member $(80 \%)$ and psychological support for PTSD $(80 \%)$. Assessment of lung function at 6 months postdischarge was endorsed by $75 \%$ of respondents.

\section{Recommendations from the free-text comments}

A total of 341 free-text comments were recorded and analysed. These informed 7 themes and 16 subthemes. See table 1 for illustrative quotes (expanded tables in online supplemental file 2).

A large proportion of the results complimented the quantitative findings; however, additional service and treatment priorities were proposed. First, respondents recognised that 'a collaborative effort for rehabilitation development' would be essential with input from experts in pulmonary/cardiac rehabilitation, nutrition, psychology, neurology, physiotherapy, respiratory medicine, occupational therapy and speech and language therapy (SALT), alongside recently published research from across the globe. Respondents felt there was a need to produce clear guidance for COVID-19 management, including this rehabilitation model, and there should be an educational campaign to promote COVID-19 rehabilitation, raise its profile among patients, carers and referrers, and embed it within the COVID-19 recovery pathway.

Second, respondents recognised the uniqueness of this pandemic and therefore highlighted the importance of continued learning from COVID-19 for service development. It was recognised this would be an iterative process as services adapt to meet the new demands and service evaluations and research develop an evidence-based model. 
Table 1 Generated themes and subthemes from the survey's free-text comments

Theme

Subthemes

A collaborative effort for rehabilitation development: to develop this model, a collaborative effort is needed from experts within the field and around the world. We can learn from international findings, current models of rehabilitation and the specialists that deliver them (eg, pulmonary/cardiac/neurological rehabilitation teams, dieticians, psychologists, respiratory consultants, respiratory and muscular skeletal physiotherapists, nurses, occupational therapists, speech and language therapists).

'I feel an effective service can only be designed if all specialists within the multi-disciplinary team are part of the development stage: physio, occupational therapist, dietitian, nurse, speech and language therapist, psychologist and any other relevant member...'

Clear guidance for COVID-19 management: there is an identified need for clear guidance and protocols for COVID-19 management, including COVID-19 rehabilitation.

A campaign to promote COVID-19 rehabilitation: it is important to raise awareness of the COVID-19 rehabilitation service across populations (service providers, referrers and patients/carers). There are suggestions to advertise it as a health promotion programme to normalise it as part of recovery on TV and radio.

Continued learning from COVID-19 for service development: it will be important to collate data for the development of the COVID-19 rehabilitation service, its evaluation and research into overall COVID-19 management. This theme acknowledges the iterative process of refining the rehabilitation service as new information comes to light and how this will inform future pandemics.

'I think we need to understand the demographics of COVID-19 survivors, as service planning for post-COVID rehab without understanding transport availability, digital literacy, ongoing psychosocial / PTSD related issues, usual working status amongst other things could result in significant oversights of what these patients are able to, and want to, engage with'.

COVID-19 patient management: overall patient management in COVID-19 recovery, including recommendations for inpatient and outpatient care.

'The 'Aftershock' isn't necessarily immediate. You can experience the euphoria of having cheated death, which may go on for some weeks/months. However, when reality hits, it can hit hard, literally overnight. Some warning that it could happen and somewhere to turn to is very important..."

Methods of rehabilitation delivery: this theme encapsulates the recommendations for how rehabilitation should be delivered and when. It is felt this is an opportunity to develop on telerehabilitation and early rehabilitation services, including adaptations and flexibility when measuring prerehabilitation and postrehabilitation outcomes.

'....programmes could be run online BUT need to ensure there is a supervised element and that access to, willingness to use and actual use are measured'.
Managing the acute phase: recommendations for inpatient care; including assessment of physical and psychological well-being to inform personalised follow-up care plans, and on discharge, the provision of a discharge bundle of assessments and advice/documentation about self-management and support for carers/family.

Early phase of recovery: recommendations for continued outpatient follow-up, including physical/psychological assessment, individualised advice on symptom management and/or referral to specialist services for additional support (eg, rehabilitation, IAPT and peer support).

Flexibility in assessment: recognising the inability to perform face-to-face consultations so adaptations to assessments are needed. Many psychometric measures can be delivered via telephone/video calls/online and alternative measures of exercise capacity can be done at home (eg, grip strength, timed up and go and sit to stand)

Early/delayed rehabilitation: there is debate about whether rehabilitation should be delivered early/later during a patient's recovery. Some respondents felt inpatient rehabilitation was appropriate, whereas others felt this would be too early for a patient's lungs and/or psychological status to have prepared for rehabilitation.

Group-based rehabilitation: safety issues inhibit group-based rehabilitation as an option currently; however, there is the option for virtual group sessions or the delivery of these once social distancing measures have relaxed. These are important for social support, especially when people are feeling isolated and alone in their recovery.

Referral and re-referral: the ability for anyone to refer to rehabilitation (eg, selfreferral and re-referral as per patient request). This needs to be a simple process which is widely known.

Telerehabilitation: this is a popular and viable option for home rehabilitation. This circumstance offers an opportunity to grow home-based rehabilitation services.

Personalised rehabilitation: the need for patient-centred rehabilitation and not a one-size-fits-all approach. There may be an opportunity to develop a multimodule rehabilitation service where modules can be selected if they are important to the patient's needs.

Components for COVID-19 rehabilitation: the components highlighted as important to a COVID-19 rehabilitation model.

Take guidance from established rehabilitation models: we should look to use/ adapt/learn from current models of rehabilitation and/or holistic care services (eg, pulmonary/cardiac/neurological/palliative/postintensive care rehabilitation, psychological support (eg, IAPT and cognitive-behavioural therapy), occupational therapy, music therapy, yoga/tai chi, SALT, community gyms, pastoral support, acupuncture and hydrotherapy).

Education, exercise and social support: the proposed components for the new rehabilitation model include

1. Education for self-management: cough, sputum clearance, breathlessness, fatigue, frailty, pain, psychologicalwell-being, behavioural change, impact of comorbidities, energy conservation, falls, improving function for daily activities, nutrition, inhaler technique, signposting, skin integrity, swallowing and voice care.

2. Exercises (physical/psychological): cognitive function, exercise programme, inspiratory muscle training and neurorehabilitation.

3. Social support: caregiver support, guidance in line with government recommendations and group activities to facilitate peer engagement. 


\section{Table 1 Continued}

\section{Theme}

A team of specialist COVID-19 rehabilitation staff: the need for an interdisciplinary team to deliver rehabilitation. They need to have been trained appropriately/have specialist skills for this patient population. '(We will need a) trained and expert team in rehabilitation medicine...'
Subthemes

Keep our staff physically safe: the need to maintain the physical health of staff who deliver rehabilitation (eg, COVID-19 testing for staff and patients, appropriate supply of personal protective equipment).

Keep our staff psychologically safe: the monitoring of staff psychological wellbeing and the provision of psychological support to support their mental health.

\section{The reassurance of financial support, recognition of the financial input and service support, is needed to develop, deliver and sustain this programme. It will need considerable financial engagement to ensure it can be rolled out nationally/internationally. \\ 'The reality of available funding and staffing post-COVID-19 pandemic should be taken into account when creating rehabilitation programmes for patients. Most services were stretched prior to the outbreak and will struggle afterwards to deliver comprehensive services for patients who are being discharged'.}

Third, alongside the early phase of recovery, suggestions for managing the acute phase were presented. Respondents highlighted the importance of assessing a patient's physical and psychological well-being to inform personalised care plans. They also wanted to see a robust COVID-19 discharge bundle of self-management materials for both patients and caregivers.

The fourth theme comprised comments relating to the appropriate methods of rehabilitation delivery. Respondents felt this was an opportunity to adapt and improve current pulmonary rehabilitation models to meet the new demands and to accommodate social distancing measures. For example, respondents suggested using preoutcome and postoutcome measures that could be assessed virtually (flexibility in assessment) using telerehabilitation with virtual group-based rehabilitation to maintain peer support. A personalised rehabilitation programme involving the assessment of patients' care needs to inform a tailored rehabilitation plan from a menu of rehabilitation modules was proposed. There was debate about the timing of rehabilitation, with some respondents leaning towards inpatient rehabilitation to minimise functional loss and others towards outpatient rehabilitation to allow time for immediate physical and psychological recovery. Access to rehabilitation was also acknowledged, with respondents highlighting the need for a clear referral pathway that healthcare professionals and patients can refer and re-refer to as necessary.

As a fifth theme, respondents proposed the necessary components for COVID-19 rehabilitation. There was acknowledgement of the effectiveness of current rehabilitation and holistic care pathways and therefore a desire not to reinvent the wheel, but rather to build on guidance from established rehabilitation models. Notably, pulmonary rehabilitation, but other suggested models of care to consider and complement might include cardiac rehabilitation, neurorehabilitation, cognitive behavioural therapy, palliative rehabilitation, SALT, music therapy, yoga/tai chi, acupuncture, pastoral support and hydrotherapy. The majority of individual components recommended for a COVID-19 rehabilitation programme mirrored the quantitative findings; however, the following topics were also presented as care priorities: sputum clearance, frailty, pain, behaviour change, the impact of comorbidities, energy conservation, falls, inhaler technique, skin integrity, swallowing and voice care, cognitive functioning, inspiratory muscle training, caregiver support, signposting and peer support through group activities.

The sixth theme identified respondents wanting to see a team of specialist COVID-19 rehabilitation staff to deliver this new model. This is to include an interdisciplinary team who has specialist skills for this patient group. Additionally, respondents felt it was important to keep our staff physically safe, for example, by ensuring an appropriate supply of personal protective equipment and the mental well-being of staff by monitoring and providing appropriate support when indicated to maintain the psychological health of the workforce.

Finally, respondents articulated the need for reassurance of financial support to ensure the robust development and delivery of this new rehabilitation model. They felt this support needed to be secured nationally to ensure equality and continuity of the service.

\section{DISCUSSION}

These data are the first from a comprehensive survey describing views from a large and diverse range of healthcare professionals about the rehabilitation needs of the post-COVID-19 population. Given the scale of response in such a short time period, there is clearly a pressing need to develop a coherent recovery programme for people who are discharged from the hospital after being infected with COVID-19. There was wide engagement with the healthcare community to support the development of the most appropriate package of rehabilitation, having secured the opinion of over 1000 respondents from a wide variety of professional backgrounds and specialties. The survey identified the important components of the immediate postdischarge phase, an assessment for a holistic rehabilitation intervention and the components of this intervention. The comments box allowed us to enrich the survey data and supported us in developing an appropriate recovery pathway for the patient postCOVID-19 (figure 4), integrating with the wider multidisciplinary team. 


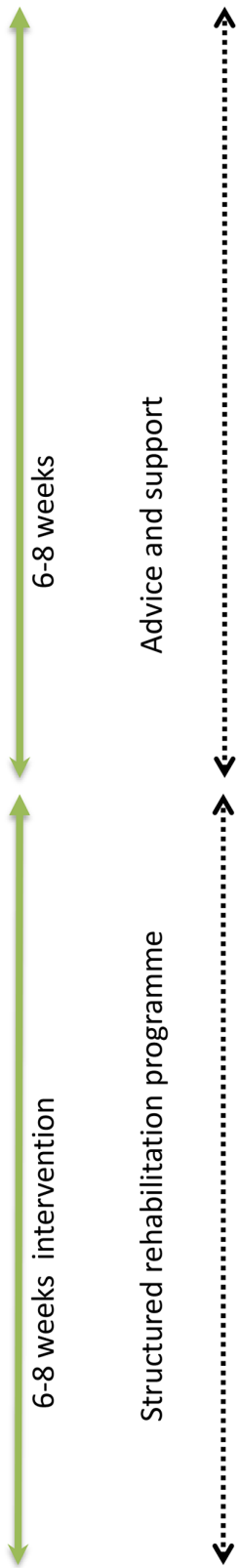

Integration Specialist

Teams e.g.

SALT

Dietitian

Physiotherapy

Occupational Therapy

Psychological Support

Services

As indicated

Figure 4 Recovery pathway for patients recovering from COVID-19. f/up, follow-up; SALT, speech and language therapy.

Pulmonary rehabilitation programmes currently deliver a personalised package of exercise and education, integrating a broad group of healthcare professionals including, but not limited to, physiotherapists, nurses, dietitians, pharmacists, psychologists, physicians, occupational therapists, exercise physiologists and graduates of the programme. As reported by respondents, there is little appetite to 'reinvent the wheel' and to develop a discreet single indication rehabilitation programme; rather there was a clear preference to adapt existing and established rehabilitation services to extend the scope to meet the needs of the post-COVID-19 population. It was clear from the comments received that this needs to be collaborative and iterative as services become more experienced to meet the demands of this 'novel' group, recognising the complex symptom burden of many recovering from COVID-19.

Many constituents recommended are already core components of a pulmonary rehabilitation pathway, assessment and programme; however, the survey gave clear guidance on the additional components required to maximise impact; these included advice and support at the time of discharge. This is an important aspect of the cardiac rehabilitation pathway after discharge from cardiac revascularisation or myocardial infarction with routine telephone follow-up. ${ }^{18}$ The advice in the early stages focuses on symptom management and returning to normal (with a focus on gentle exercise and employment/financial issues). 
The assessment of the patient post-COVID-19 at 6-8 weeks requires a much broader approach than commonly adopted by pulmonary programmes, specifically screening for PTSD and fatigue as a discreet symptom. PTSD is reported as a core outcome measure in the consensus statement for the follow-up of ICU survivors. ${ }^{19}$ This report indicated that consensus was achieved for measures of mood, quality of life and PTSD, while exercise capacity and cognition almost reached consensus. It is beyond the scope of this survey to indicate the most appropriate outcome measures for the rehabilitation of the postCOVID-19 population, but there would seem a great deal of logic in combining the core measures of pulmonary rehabilitation with the outcomes recommended in the post-ICU population. Interestingly the conduct of a face-to-face assessment was recommended by $68 \%$, circumstances where face-to-face assessments are challenging and require adherence to strict infection control processes. This is reflected in the free-text comments with respect to service users' and providers' safety concerns. Additional comments from the survey identified the importance of measuring cognition and, importantly, the need for integration with social care and SALT. The timing and the modes of delivery were a discreet theme, and include issues such as the feasibility of a face-to-face assessment and the need to be flexible in the current environment, with digital/telehealth solutions being highlighted as options. Moving forward, digital solutions may be important to increase capacity and give patients a choice. ${ }^{20}$ A subtheme arose, identifying the need for clear guidance. Previous international literature has described pulmonary rehabilitation services supporting recovery in other respiratory epidemics (SARS), ${ }^{4}$ but the survey rightly reflects the need to collaborate with a much wider interdisciplinary team to offer the best service to patients post-COVID-19. There is now emerging evidence from national and international societies stating a pulmonary rehabilitation framework will need to be adapted to suit the recovery needs of the individual with COVID-19. ${ }^{21} 22$

This work highlights the real need for rehabilitating the post-COVID-19 population and is strengthened by the large number of respondents; however, we acknowledge a large proportion of these were physiotherapists and female, which limits our scope for generalising the results to all relevant specialties. Furthermore, 25\% of respondents had no experience in managing patients with COVID-19, and $31 \%$ had no experience in rehabilitation. Additionally, we did not consider the views of patients, carers or the public; this is currently being undertaken by the British Lung Foundation. ${ }^{23}$ These two surveys taken together should support guidance on the provision of rehabilitation services for the patient post-COVID-19.

It would seem that there is a real opportunity to develop a structured interdisciplinary rehabilitation programme that addresses the complex needs of the post-COVID-19 population, including those who had a period on ICU through to those managed in the community. The provision of post-ICU rehabilitation, although recommended, ${ }^{24}$ is poorly provided.$^{25} \mathrm{~A}$ potential and desirable legacy of this pandemic is to raise the provision of post-ICU care. However, it is important that capacity development is supported, in order to not compromise the service for those who routinely access these established rehabilitation programmes.

However, the more immediate challenge is to deliver a recovery pathway for those individuals who are being discharged now and for all those who have been discharged over the last few weeks with a diagnosis of COVID-19. We should use these survey data to inform service delivery and work collaboratively across specialties and professions to deliver a comprehensive recovery package for the COVID-19 population while of course retaining the high quality of service delivered to the usual case load of individuals with chronic respiratory disease.

\section{CONCLUSION}

These data on over 1000 respondents reflect the interest in the field of rehabilitation and the urgent need to adapt existing services to meet the complex set of needs of patients with COVID-19. Overall, there was a high level of agreement for the components of an early intervention, the elements of assessment and the components of the subsequent rehabilitation programme. This pandemic presents a real opportunity for truly collaborative working across disciplines and specialties and should be an immediate priority to mitigate the long-term impact of COVID-19.

\section{Twitter Amy C Barradell @AmyBarradell and Charlotte Bolton @bolton_char}

Acknowledgements The authors thank the British Thoracic Society (particularly Sheila Edwards, Sally Welham and Kathryn Wilson) for supporting the concept of the survey, developing the online survey, distributing to members and supporting the analysis. Furthermore, the authors thank all the participants and those who promoted the survey, allowing the achievement of the high response rate.

Contributors SJS, NJG, CB GJ and JRH contributed to the conception and design of the study. LP, ACB and SJS analysed the data. SJS and ACB interpreted the data and drafted the manuscript. All authors critically revised the manuscript for significant intellectual content and insight, had full access to all of the data, and take responsibility for the integrity and accuracy of the data analysis. In addition, all authors gave final approval of the manuscript version for publication. SJS and ACB were responsible for the overall content as guarantors.

Funding The authors have not declared a specific grant for this research from any funding agency in the public, commercial or not-for-profit sectors.

Competing interests All authors have completed the Unified Competing Interests Form at http://www.icmje.org.coi_disclosure.pdf. SJS reports grants from Actegy and Pfizer outside the submitted work. GJ reports grants from Astra Zeneca, Biogen, Galecto and GlaxoSmithKline; personal fees from Boehringer Ingelheim, Daewoong, Galapagos, Heptares, Promedior and Roche; non-financial support from NuMedii; grants and personal fees from Pliant; non-financial support from Redx; and others from Action for Pulmonary Fibrosis outside the submitted work.

\section{Patient consent for publication Not required.}

Ethics approval Ethical approval was not required for this survey from either the UK Health Research Authority or leading research and development centre. Completion of the survey was an indication of willingness to participate and implied consent. We set no threshold for response over such a short period of time but were anticipating around 300 responses across a range of healthcare professionals to allow the questionnaire to be considered robust and representative of those in the field.

Provenance and peer review Not commissioned; externally peer reviewed. 
Data availability statement All data relevant to the study are included in the article or uploaded as supplementary information. No additional data are available.

Supplemental material This content has been supplied by the author(s). It has not been vetted by BMJ Publishing Group Limited (BMJ) and may not have been peer-reviewed. Any opinions or recommendations discussed are solely those of the author(s) and are not endorsed by BMJ. BMJ disclaims all liability and responsibility arising from any reliance placed on the content. Where the content includes any translated material, BMJ does not warrant the accuracy and reliability of the translations (including but not limited to local regulations, clinical guidelines, terminology, drug names and drug dosages), and is not responsible for any error and/or omissions arising from translation and adaptation or otherwise.

Open access This is an open access article distributed in accordance with the Creative Commons Attribution Non Commercial (CC BY-NC 4.0) license, which permits others to distribute, remix, adapt, build upon this work non-commercially, and license their derivative works on different terms, provided the original work is properly cited, appropriate credit is given, any changes made indicated, and the use is non-commercial. See: http://creativecommons.org/licenses/by-nc/4.0/.

ORCID iDs

Amy C Barradell http://orcid.org/0000-0002-3688-8879

John R Hurst http://orcid.org/0000-0002-7246-6040

\section{REFERENCES}

1 Huang C, Wang Y, Li X, et al. Clinical features of patients infected with 2019 novel coronavirus in Wuhan, China. Lancet 2020;395:497-506.

2 Wang D, Hu B, Hu C, et al. Clinical characteristics of 138 hospitalized patients with 2019 novel coronavirus-infected pneumonia in Wuhan, China. JAMA 2020;323:1061-9.

3 Wang L, He W, Yu X, et al. Coronavirus disease 2019 in elderly patients: characteristics and prognostic factors based on 4-week follow-up. J Infect 2020;80:639-45.

4 Hui DSet al. Impact of severe acute respiratory syndrome (SARS) on pulmonary function, functional capacity and quality of life in a cohort of survivors. Thorax 2005;60:401-9.

5 Luyt C-E, Combes A, Becquemin M-H, et al. Long-term outcomes of pandemic 2009 influenza $A(\mathrm{H} 1 \mathrm{~N} 1)$-associated severe ARDS. Chest 2012;142:583-92.

6 Crisafulli E, Gorgone P, Vagaggini B, et al. Efficacy of standard rehabilitation in COPD outpatients with comorbidities. Eur Respir J 2010;36:1042-8

7 Vanfleteren LEGW, Spruit MA, Groenen M, et al. Clusters of comorbidities based on validated objective measurements and systemic inflammation in patients with chronic obstructive pulmonary disease. Am J Respir Crit Care Med 2013;187:728-35.
8 Steiner M, McMillan V, Lowe D, et al. National COPD audit programme pulmonary rehabilitation: an exercise in improvement, 2018. Available: www.rcplondon.ac.uk/nacap

9 Zhou F, Yu T, Du R, et al. Clinical course and risk factors for mortality of adult inpatients with COVID-19 in Wuhan, China: a retrospective cohort study. Lancet 2020;395:1054-62.

10 Guan W-J, Liang W-H, He J-X, et al. Cardiovascular comorbidity and its impact on patients with COVID-19. Eur Respir J 2020;55:2001227.

11 Greenhalgh T, Knight M, A'Court C, et al. Management of post-acute covid-19 in primary care. BMJ 2020;370:m3026.

12 Steiner M, Holzhauer-Barrie J, Lowe D, et al. National COPD audit programme: pulmonary rehabilitation: time to breathe better, 2015.

13 Docherty AB, Harrison EM, Green CA, et al. Features of 16,749 hospitalised UK patients with COVID-19 using the ISARIC who clinical characterisation protocol. medRxiv 2020.

14 Azoulay E, Vincent J-L, Angus DC, et al. Recovery after critical illness: putting the puzzle together-a consensus of 29. Crit Care 2017;21:296.

15 Hsieh M-J, Lee W-C, Cho H-Y, et al. Recovery of pulmonary functions, exercise capacity, and quality of life after pulmonary rehabilitation in survivors of ARDS due to severe influenza $A(H 1 N 1)$ pneumonitis. Influenza Other Respir Viruses 2018;12:643-8.

16 ERS. In the line of duty | European respiratory Society. Available: https://www.ersnet.org/covid-19-blog [Accessed 4 May 2020].

17 Braun V, Clarke V. Using thematic analysis in psychology. Qual Res Psychol 2006;3:77-101.

18 Dalal HM, Doherty P, Taylor RS. Cardiac rehabilitation. BMJ 2015;351:h5000.

19 Needham DM, Sepulveda KA, Dinglas VD, et al. Core outcome measures for clinical research in acute respiratory failure survivors. An international modified Delphi consensus study. Am J Respir Crit Care Med 2017;196:1122-30.

20 NHS England. NHS to offer 'long covid' sufferers help at specialist centres, 2020. Available: https://www.england.nhs.uk/2020/10/nhsto-offer-long-covid-help/ [Accessed 20 Oct 2020].

21 Singh SJ, Bolton C, Nolan C, et al. Delivering rehabilitation to patients surviving COVID-19 using an adapted pulmonary rehabilitation approach - BTS guidance, 2020. Available: https:// www.brit-thoracic.org.uk/document-library/quality-improvement/ covid-19/pulmonary-rehabilitation-for-covid-19-patients/

22 Spruit MA, Holland AE, Singh SJ, et al. COVID-19: interim guidance on rehabilitation in the hospital and Post-Hospital phase from a European respiratory Society and American thoracic Societycoordinated international Task force. Eur Respir J 2020:2002197.

23 UK. Post-COVID survey, 2020. Available: https://auk2016.typeform. com/to/LIhQXR [Accessed 7 May 2020].

24 National Institute for Heath and Care Excellence. Rehabilitation after critical illness in adults. Natl Inst Heal Care Excell 2009:1-27.

25 Connolly B, Douiri A, Steier J, et al. A UK survey of rehabilitation following critical illness: implementation of NICE clinical guidance 83 (CG83) following hospital discharge. BMJ Open 2014;4:4963. 